\title{
Nomograms for predicting overall survival and cancer-specific survival in young patients with pancreatic cancer in the US based on the SEER database
}

\author{
Min Shi ${ }^{\text {Equal first author, 1 , Biao Zhou }}{ }^{\text {Equal first author, } 1}$, Shu-Ping Yang ${ }^{\text {Corresp. } 1}$ \\ ${ }^{1}$ Department of Gastroenterology, Liyang Branch of Jiangsu Province Hospital, Liyang, China \\ Corresponding Author: Shu-Ping Yang \\ Email address: shupingy@163.com
}

Background: The incidence of young patients with pancreatic cancer (PC) is on the rise, and there is a lack of models that could effectively predict their prognosis. The purpose of this study was to construct nomograms for predicting the overall survival (OS) and cancer-specific survival (CSS) of young patients with PC.

Methods: PC patients younger than 50 years old from 2004 to 2015 in the Surveillance, Epidemiology, and End Results (SEER) database were selected and randomly divided into training set and validation set. Univariate and forward stepwise multivariate Cox analysis was used to determine the independent factors affecting OS. Fine and Gray competing risk regression model was used to determine the independent factors affecting CSS. We used significant variables in the training set to construct nomograms predicting prognosis. The discrimination and calibration power of models were evaluated by concordance index (C-index), calibration curve and 10-flod cross-validation.

Results: 4,146 patients were selected. Multivariate Cox analysis showed that gender, race, grade, pathological types, AJCC stage and surgery were independent factors affecting OS. The C-index of the nomogram predicting OS in training and validation was 0.733 (average $=0.731,95 \% \mathrm{Cl}=0.724-0.738$ ) and $0.742(95 \% \mathrm{Cl}=0.725-0.759)$, respectively. Competing risk analysis showed that primary site, pathological types, AJCC stage and surgery were independent factors affecting CSS. The C-index of the nomogram predicting CSS in training and validation set was 0.792 (average $=0.765,95 \% \mathrm{Cl}=0.742-0.788$ ) and 0.776 $(95 \% \mathrm{Cl}=0.773-0.779)$, respectively. C-index based on nomogram was better in training and validation set than that based on AJCC stage. Calibration curves showed that these nomograms could accurately predict the 1-, 3- and 5-year OS and CSS both in training set and validation set.

Conclusions: The nomograms could effectively predict OS and CSS in young patients with PC, which help clinicians more accurately and quantitatively judge the prognosis of individual patients. 
1 Nomograms for predicting overall survival and cancer-specific survival in young patients with

2 pancreatic cancer in US based on the SEER database

3 Min Shi ${ }^{1, \#}$, Biao Zhou ${ }^{1, \#}$, Shu-ping Yang ${ }^{1}$

4 Authors' affiliations:

5 1: Department of Gastroenterology, Liyang Branch of Jiangsu Province Hospital, Jianshe west

6 Road No. 70, Liyang, China

7 \#: These authors contributed equally to this work and should be considered co-first authors.

8 Corresponding author: Shu-ping Yang, E-male: shupingy@163.com, phone number: +86-

$9 \quad 13813900689$

10 Conflicts of interest: The authors declare they have no conflict of interest

11 Abstract

12 Background: The incidence of young patients with pancreatic cancer (PC) is on the rise, and

13 there is a lack of models that could effectively predict their prognosis. The purpose of this study

14 was to construct nomograms for predicting the overall survival (OS) and cancer-specific survival

15 (CSS) of young patients with PC.

16 Methods: PC patients younger than 50 years old from 2004 to 2015 in the Surveillance,

17 Epidemiology, and End Results (SEER) database were selected and randomly divided into

18 training set and validation set. Univariable and forward stepwise multivariable Cox analysis was

19 used to determine the independent factors affecting OS. Fine and Gray competing risk regression

20 model was used to determine the independent factors affecting CSS. We used significant 
21

variables in the training set to construct nomograms predicting prognosis. The discrimination and calibration power of models were evaluated by concordance index (C-index), calibration curve and 10-flod cross-validation.

Results: 4,146 patients were selected. Multivariable Cox analysis showed that gender, race, grade, pathological types, AJCC stage and surgery were independent factors affecting OS. The C-index of the nomogram predicting OS in training and validation was 0.733 (average $=0.731,95 \% \mathrm{CI}=0.724-0.738)$ and $0.742(95 \% \mathrm{CI}=0.725-0.759)$, respectively. Competing risk analysis showed that primary site, pathological types, AJCC stage and surgery were independent factors affecting CSS. The C-index of the nomogram predicting CSS in training and validation set was 0.792 (average $=0.765,95 \% \mathrm{CI}=0.742-0.788)$ and $0.776(95 \% \mathrm{CI}=0.773-0.779)$, respectively. C-index based on nomogram was better in training and validation set than that based on AJCC stage. Calibration curves showed that these nomograms could accurately predict the 1-, 3- and 5-year OS and CSS both in training set and validation set.

Conclusions: The nomograms could effectively predict OS and CSS in young patients with PC, which help clinicians more accurately and quantitatively judge the prognosis of individual patients.

Keywords: younger pancreatic cancer, SEER, nomograms, prognosis

Introduction

PC is a highly malignant tumor. Global cancer data show that PC ranks 14 th and 7 th among all cancers in terms of incidence and mortality, respectively.[1] It is estimated that by 2030, the 
41 mortality rate of PC will be the second highest in the world.[2] According to the American

42 Cancer Society, in 2019, the United States will have 56,770 new cases of PC and 45,750 will die

43 of PC. The 5-year survival rate of PC is the lowest of all cancers, with only $9 \%$.[3]

44 The incidence of PC in young patients is on the rise. A survey based on the SEER database

45 found that the incidence of PC among young whites and blacks increased by 57\% between 2001

46 and 2015.[4] In addition, another epidemiological study found that from 1992 to 2013, the

47 annual percentage change in the incidence of PC in women aged 25-34 was more than 2.5\%.[5]

48 The median age of diagnosis of PC is 71 years old.[6] Due to ethnic and regional differences, the

49 definition of " young patients " with PC has not been unified. Previous studies have generally

50 classified young PC at the age of 45[7-9] or 50[10-12]. In this study, we screened PC patients

51 younger than 50 years old from the SEER database in order to enable more people to benefit

52 from our study.

53 American Joint Committee on Cancer (AJCC) stage has always provided a reference for judging

54 the progress of the disease and for the choice of clinical decision-making. However, the

55 researchers found that the staging system is insufficient .[13-15] It only incorporates some

56 features of the tumors into the staging system, and the prognostic factors are far more than these.

57 Previous studies have found that age, sex, grade, different treatment and even marital status are

58 important independent factors affecting the prognosis of patients with PC.[16-19]

59 A nomogram is a graphical calculation or algorithm that combines several continuous variables

60 to predict a specific end point using traditional statistical methods (for example, Logistic or Cox 
61 regression model).[20] It has been constructed and verified in a variety of cancers to help

62 clinicians quickly and accurately judge the prognosis of patients.[17, 21, 22] Many studies[7-12,

63 23] have confirmed that the clinical characteristics and prognosis of young patients with PC are

64 different from older patients, but the nomogram of PC in previous studies[17, 24, 25] cannot

65 accurately predict the prognosis of young patients with PC.

66 The aim of this study was to establish and validate nomograms to predict 1-, 3-, and 5-year OS

67 and CSS based on data from younger PC patients in the SEER database between 2004 and 2015.

68 Patients and methods

69 Selection of patients

70 PC patients younger than 50 years old who were diagnosed from January 1, 2004 to December 31,

712015 were selected from the Incidence-SEER 18 Registries Custom Data (with additional

72 treatment fields), released April 2019, based on the November 2018 submission. The patient meted

73 the following conditions: (1) site recode ICD-0-3/WHO 2008:"pancreas"; (2) positive histology;

74 (3) active follow-up; (4) one primary only. The exclusion criteria were as follows: (1) age, race,

75 surgery unknown; (2) AJCC stage NOS, N/A or unknown; (3) age at diagnosed $\geqslant 50$ years old;

76 (4) survival time unknown and less than 1 month. The detailed flow chart was shown in Figure 1.

77 For this study, we signed the SEER research data agreement to access SEER information with the

78 username10067-Nov2018. The SEER database is publicly available and the data for all patients

79 are de-identified, so the approval and informed consent of the institutional review committee were

80 not required in the current study. 
81 Variable Classification

82 clinical variables including race, gender, primary site, grade, pathological types, AJCC stage,

83 status of surgery, survival time, status of survival and cause for death were extracted from the

84 SEER database. The degree of differentiation of tumors was divided into three groups: grade I

85 (well differentiated) and grade II (moderately differentiated) were high differentiated, grade III

86 (poorly differentiated) and grade IV (undifferentiated) were low differentiated, and unknown.

87 The pathological types were divided into ductal adenocarcinoma and non-ductal

88 adenocarcinoma. The International Classification of Diseases for Oncology, Third Edition (ICD-

89 0-3) codes of ductal adenocarcinoma were 8500 and 8140.[26] The staging of cancer is based on

90 the 6th edition of AJCC stage, which adapted to patients in the SEER database with a diagnosis

91 time of 2004-2015.

92 Statistical Analysis

93 In this study, the whole cohort was randomly divided into two groups, 2,904 (70\%) were training

94 set and 1,242 (30\%) were validation set. Chi-square test was used to compare the

95 clinicopathological characteristics between the training set and the validation set.

96 OS referred to the duration from diagnosis to any original death or last follow-up. variables

97 associated with OS in univariable analysis $(\mathrm{p}<0.05)$ were included into multivariable analysis.

98 The method of forward stepwise selection in a multivariable regression model was applied to the

99 training cohort to select variables. The independent prognostic factors on multivariable analysis

100 were used to construct nomogram for OS. 
101 Considering that death from other causes is a competitive event of pancreatic cancer death, we

102 constructed a competing risk nomogram to predict CCS. CSS referred to the duration from

103 diagnosis to death from PC, patients who were alive at the point of last follow-up were

104 considered as censored events. Variables related with CSS $(\mathrm{p}<0.05)$ in the univariable analysis

105 or with important clinical value were included into a multivariable analysis based on

106 proportional subdistribution hazard models and those independent variables were selected to

107 build a nomogram for CSS.

108 Discrimination of the nomograms was measured through the concordance index (C-index) with

109 its respective 95\% confidence interval (CI), which quantifies the level of concordance between

110 probabilities of prediction and the actual chance of having the event of interest.[27] The larger

111 the C-index is, the more accurate the nomogram is to predict the prognosis.[28]10-fold cross-

112 validation was applied to verify the stability of the model and calculate the average value of $\mathrm{C}$

113 index in the training group. In order to reduce the overfit bias, calibration was evaluated by

114 comparing the actual probabilities and the plot of the nomogram using 1000 bootstrap samples.

115 In the calibration curve, the vertical axis is the actual value and the horizontal axis is the

116 predicted value. If the actual / predicted value passes through the origin along the $45^{\circ}$ line, it

117 shows that the nomogram has been well calibrated.[27]

118 For all analyses, p-value $<0.05$ was considered statistically significant. All date was obtained

119 through SEER*Stat software version 8.3.5. Fine and Gray competing risk Statistical analyses 
120 were performed using R work, and the others performed by SPSS (IBM, NY). The nomograms

121 and calibration curves were draw using R version 3.6.0 (http://www.r-project.org)

122 Results

123 Patients characteristic

124 Finally, there were a total of 4,146 cases of young patients with PC diagnosed in the SEER

125 database between January 1, 2004 and December 31, 2015. In the entire cohort, in terms of

126 demography, mainly whites (74.1\%) and males (53.7\%); In terms of tumor characteristics,

127 pancreatic head cancer $(46.1 \%)$ was the most common, more highly differentiated tumors

128 (30.7\%), mainly ductal adenocarcinoma (68.0\%), in addition, more advanced tumors in diagnosis

129 (55.7\%); In terms of treatment, the proportion of patients undergoing surgery was low (37.5\%).

130 Detailed patient clinical characteristics were summarized in Table 1.

131 Construction and validation of nomogram for predicting OS of young patients with PC

132 The results of univariable and multivariable Cox regression models for OS were shown in Table

133 2. Univariable analysis showed that race, gender, primary site, grade, pathological types, AJCC

134 stage and surgery were correlated with OS $(\mathrm{p}<0.05)$. Multivariable analysis suggested that

135 gender, grade, pathological types, AJCC stage and surgery were independent risk factors for OS.

136 In details, female (HR:0.91, 95\% $\mathrm{CI}=0.83-0.98 ; \mathrm{p}=0.019)$, non-ductal adenocarcinoma

137 (HR:0.40,95\%CI=0.36-0.45; $\mathrm{p}<0.001)$ and receiving surgery $(\mathrm{HR}: 0.43,95 \% \mathrm{CI}=0.38-0.50$;

$138 \mathrm{p}<0.001)$ had a better OS. Black $(\mathrm{HR}: 1.15,95 \% \mathrm{CI}=1.03-1.28 ; \mathrm{p}=0.016)$, low differentiated

139 tumor $(\mathrm{HR}: 1.59,95 \% \mathrm{CI}=1.40-1.80 ; \mathrm{p}<0.001)$ and advanced stage (HR:4.63, 95\% $\mathrm{CI}=3.53-6.07$; 
$140 \mathrm{p}<0.001)$ had a worse OS. The Nomogram was constructed of the above six variables in training

141 set (Figure 2.A). It can be seen from the nomogram that AJCC stage, which range of risk score is

142 from 0 to 100, had the greatest contribution on OS (including 1- ,3- and 5-years), followed by

143 pathological subtypes, surgery, grade, race and gender. The detailed steps for the application of

144 the nomogram are as follows: Draw a vertical line to the horizontal axis marked "points" at the

145 top of the nomogram according to the classification (e.g., gender is divided into male and

146 female) of each prognostic variable (gender, grade, pathological type, AJCC stage, and surgery).

147 At the position where the vertical line passes through the "Points" axis, each prognostic variable

148 can obtain a score. Add the scores of the five variables to get the total score, find the position of

149 the total score on the horizontal axis marked as "total points", and draw a vertical line from the

150 total score position marked on the horizontal axis of "Total Points" to the 1 -, 3-and 5-years OS

151 axis. Where the vertical line intersects the 1-year OS axis is the \% probability of the 1-year

152 overall survival.

153 Compared with C-index based on AJCC stage in training set $(0.677,95 \% \mathrm{CI}=0.666-0.688)$ and

154 validation set $(0.672,95 \% \mathrm{CI}=0.656-0.688)$, The $\mathrm{C}$-index of our model in the training set and

155 validation set was 0.733 (average $=0.731,95 \% \mathrm{CI}=0.724-0.738)$ and $0.742(95 \% \mathrm{CI}=0.725-0.759)$

156 respectively, showing a better degree of discrimination. The calibration curve of training set and

157 validation set showed good consistency between prediction and observation in the probability of

1581 -, 3 -, and 5-year OS, respectively (Figure 3).

159 Construction and validation of nomogram for predicting CSS of young patients with PC 
160 The results of univariable and multivariable competing risks models for CSS were shown in

161 Table 3. Fine and Gray analysis showed that primary site, grade, pathological types, AJCC stage

162 and surgery were correlated with CSS. Multivariable analysis suggested that primary site,

163 pathological types, AJCC stage and surgery were independent risk factors for CSS. In details,

164 non-ductal adenocarcinoma (SHR:0.57, 95\%CI=0.50-0.65; $<<0.001)$, receiving surgery

165 (SHR:0.77, 95\%CI=0.68-0.87; $<<0.001)$ and tumors in others site (SHR:0.87, 95\%CI=0.77-0.99;

$166 \mathrm{p}=0.037)$ had a better CSS. Advanced stage $(\mathrm{SHR}: 1.53,95 \% \mathrm{CI}=1.22-1.93 ; \mathrm{p}<0.001)$ had a worse

167 CSS. The Nomogram was constructed of the above four variables in training set (Figure2.B). It

168 also can be seen from the nomogram that AJCC stage had the greatest effect on CSS (including

169 1-,3- and 5-years), followed by pathological subtypes, surgery and primary site. Finally, similar

170 to the above, we could also predict 1 -, 3 -, and 5-year CSS in patients with PC.

171 Compared with C-index based on AJCC stage in training set $(0.706,95 \%, \mathrm{CI}=0.704-0.707)$ and

172 validation set $(0.692,95 \% \mathrm{CI}=0.695-0.689)$, The $\mathrm{C}$-index of our model in the training set and

173 validation set was 0.792 (average $=0.765,95 \% \mathrm{CI}=0.742-0.788)$ and $0.776(95 \% \mathrm{CI}=0.773-0.779)$,

174 respectively, showing a better degree of discrimination. The calibration curve of training set and

175 validation set also showed good consistency between prediction and observation in the

176 probability of 1 -, 3 -, and 5-year CSS, respectively (Figure 4).

177 Discussion

178 In this study, we determined that race, gender, grade, pathological types, surgery and AJCC stage

179 were independent factors affecting the OS of younger patients with PC by univariable and 
180

181

182

183

184

185

186

187

188

189

190

191

192

193

194

195

196

197

198

199

multivariable regression analysis based on the SEER database, and competing risk analysis was

used to determine that surgery, pathological types and AJCC stage and primary site were

independent factors affecting CSS in younger patients with PC. We integrated the factors and

draw nomograms that could effectively predict $1-, 3$-, and 5-year OS and CSS in younger

patients with PC.

As far as we know, this was the first time that a nomogram based on a large multicenter dataset has been constructed to effectively predict the prognosis of younger patients with PC. Previous studies[17, 24, 25, 29] found that age was an independent factor affecting the OS or CSS of patients with $\mathrm{PC}$, and most of them divided age into $<60$ years (or 65 years) and $\geq 60$ years (or 65 years). There were differences in clinicopathological characteristics and prognosis between young patients with PC and elderly patients.[7-12, 23] Our data showed that primary site was an independent factor affecting CSS in young patients with PC, which was consistent with previous study.[24] Another study[29] used five variables, including age, differentiation, TNM staging, surgery and lymph node surgery, to construct a nomogram for predicting the OS rate of patients with PC, of which age was divided into $25-39,40-59,60-79$ and $80+$. But the study still had some limitations. They included only patients with non-metastasis and ductal adenocarcinoma. Our study found that in young patients with PC, patients with distant metastasis and non-ductal adenocarcinoma accounted for about $55.7 \%$ and $32.0 \%$ of the total population, respectively. Therefore, it was necessary to establish a nomogram that could predict the prognosis of young patients with PC. 
200 In our study, AJCC stage and surgery were independent factors affecting OS and CSS in young

201 patients with PC, which was consistent with the results of previous studies[17, 24, 29] in patients

202 with PC. AJCC stage had the greatest influence on OS and CSS in young patients with PC,

203 followed by pathological type, surgery and grade. Additionally, it was worth noting that the

204 impact of pathological types on prognosis was even greater than that of surgery. Mohamed E.

205 Mostafa et al[30] pointed out that although ductal adenocarcinoma was the most common

206 pathological type of PC, there were many other pathological types of PC in the real world, such

207 as solid pseudopapillary tumors, neuroendocrine tumors, and so on. They had different

208 clinicopathological characteristics and biological behavior. Therefore, more research was needed

209 to focus on non-ductal adenocarcinoma. There were racial differences in the OS of young

210 patients with PC, and the risk of death was higher in blacks. This might be due to the higher

211 incidence of distant pancreatic cancer in black patients.[4]

212 Compared with the traditional AJCC stage, all the C-index of our model were more than 0.72,

213 indicating a better discrimination and the ability to provide individualized prediction for patients.

214 For example, two patients with stage II PC after surgery, one was a male with low differentiated

215 ductal adenocarcinoma, and the other was a female with high differentiated non-ductal

216 adenocarcinoma. According the Table S1, the 1-year OS of the two patients could be calculated

217 to be about $56 \%$ and $88 \%$, respectively. However, according to the AJCC stage, both of them

218 were patients with stage II PC, and it was difficult to compare the differences in prognosis

219 between them. 
220 Our research had some advantages. First of all, our study was based on the SEER database,

221 which covered $28 \%$ of the population of the United States, so the nomograms were more

222 applicable. Secondly, compared with the previous nomograms used to evaluate the prognosis of

223 PC patients, our models were more targeted to evaluate the prognosis of PC patients younger

224 than 50 years old. Finally, the calibration curves of our training set and validation set showed

225 good consistency, indicating that our nomograms had good calibration power.

226 Even so, some limitations still existed in our study. First, at present, surgery was still the main

227 treatment for patients with PC, but studies have found that radiotherapy and chemotherapy can

228 also effectively improve the prognosis of postoperative patients with PC.[31, 32] Detailed

229 radiotherapy or chemotherapy regimens were not available from the SEER database. In addition,

230 other known risk factors for prognosis, such as family history[33], tobacco or alcohol[34] were

231 also hard to obtain from the SEER database. Moreover, in this study, patients were randomly

232 divided into two groups, $70 \%$ of them were used to construct and other $30 \%$ were used to

233 validate the nomograms. Although both the $\mathrm{C}$ index and the calibration curve performed well,

234 external validation was still needed in other populations to evaluate the accuracy of our models.

235 Conclusion

236 We developed and validated nomograms that could effectively predict the prognosis of PC

237 patients younger than 50 years old. These nomograms could help clinicians more accurately and

238 conveniently predict the 1-, 3-and 5-year OS and CSS of individual patients.

239 Abbreviation:

Peer) reviewing PDF | (2019:09:41386:2:0:NEW 29 Feb 2020) 
240 PC: pancreatic cancer

241 OS: overall survival

242 CSS: cancer-specific survival

243 SEER: Surveillance, Epidemiology, and End Results

244 C-index: concordance index

245 AJCC: American Joint Committee on Cancer

246 Figure legends

247 Figure 1. Flowchart of patient selection.

248 Figure1 contains detailed selection of PC patients in 2004-2015 from SEER database.

249 Figure 2. Nomogram for predicting OS and CSS of young patients with PC.

250 Figure 2 contains: A. Nomogram for predicting 1-, 3- and 5-year OS for young patients with PC;

251 B. Nomogram for predicting 1-, 3- and 5-year CSS for young patients with PC

252 Figure 3. Calibration curves for 1-, 3- and 5-year OS in training set and validation set.

253 Figure 3 contains: A. Calibration curves for 1-year OS in training set; B. Calibration curves for

254 3-year OS in training set; C. Calibration curves for 5-year OS in training set; D. Calibration

255 curves for 1-year OS in validation set; E. Calibration curves for 3-year OS in validation set; F.

256 Calibration curves for 5-year OS in validation set.

257 Figure 4. Calibration curves for 1-, 3- and 5-year CSS in training set and validation set.

258 Figure 4 contains: A. Calibration curves for 1-year CSS in training set; B. Calibration curves for

259 3-year CSS in training set; C. Calibration curves for 5-year CSS in training set; D. Calibration 
260 curves for 1-year CSS in validation set; E. Calibration curves for 3-year CSS in validation set; F.

261 Calibration curves for 5-year CSS in validation set.

262 Acknowledgments

263 The authors acknowledge the efforts of the Surveillance, Epidemiology, and End Results

264 Program tumor registries in the creation of the SEER database.

265 Reference

266 1. Bray, F., et al., Global cancer statistics 2018: GLOBOCAN estimates of incidence and

267 mortality worldwide for 36 cancers in 185 countries. CA Cancer J Clin, 2018. 68(6): p. 394-

268

269 424.

2. Rahib, L., et al., Projecting cancer incidence and deaths to 2030: the unexpected burden

270

271

272

273

274

275

276

277

278

279

6. Midha, S., S. Chawla, and P.K. Garg, Modifiable and non-modifiable risk factors for

280

281

7. Bunduc, S., et al., Very Early Onset Pancreatic Adenocarcinoma-Clinical Presentation, Risk

282

283 of thyroid, liver, and pancreas cancers in the United States. Cancer Res, 2014. 74(11): p. 2913-21.

3. Siegel, R.L., K.D. Miller, and A. Jemal, Cancer statistics, 2019. CA Cancer J Clin, 2019. 69(1):

4. Tavakkoli, A., et al., Racial Disparities and Trends in Pancreatic Cancer Incidence and

5. Gordon-Dseagu, V.L., et al., Pancreatic cancer incidence trends: evidence from the Surveillance, Epidemiology and End Results (SEER) population-based data. Int J Epidemiol, 2018. 47(2): p. 427-439.

10. Ntala, C., et al., Demographic, clinical, and pathological features of early onset pancreatic Factors and Therapeutic Options. Chirurgia (Bucur), 2018. 113(3): p. 405-411.

8. Kang, J.S., et al., Clinicopathologic and survival differences in younger patients with pancreatic ductal adenocarcinoma-A propensity score-matched comparative analysis. Pancreatology, 2017. 17(5): p. 827-832.

9. He, J., et al., Young patients undergoing resection of pancreatic cancer fare better than their older counterparts. J Gastrointest Surg, 2013. 17(2): p. 339-44.

290

11. Ansari, D., et al., Early-onset pancreatic cancer: a population-based study using the SEER

12. Beeghly-Fadiel, A., et al., Early onset pancreatic malignancies: Clinical characteristics and 
293 survival associations. Int J Cancer, 2016. 139(10): p. 2169-77.

294 13. Adam, M.A., et al., Rethinking the Current American Joint Committee on Cancer TNM

295

296

297

298

299

300

301

302

303

304

305

306

307

308

309

310

311

312

313

314

315

316

317

318

319

320

321

322

323

324

325

326

327

328

329

330

331 Staging System for Medullary Thyroid Cancer. JAMA Surg, 2017. 152(9): p. 869-876.

14. Choi, K.H., et al., Comparison the sixth and seventh editions of the AJCC staging system for T1 gastric cancer: a long-term follow-up study of 2124 patients. Gastric Cancer, 2017. 20(1): p. 43-48.

15. Shao, N., et al., Comparison of the 7th and 8th edition of American Joint Committee on Cancer (AJCC) staging systems for breast cancer patients: a Surveillance, Epidemiology and End Results (SEER) Analysis. Cancer Manag Res, 2019. 11: p. 1433-1442.

16. Li, H.B., J. Zhou, and F.Q. Zhao, A Prognostic Nomogram for Disease-Specific Survival in Patients with Pancreatic Ductal Adenocarcinoma of the Head of the Pancreas Following Pancreaticoduodenectomy. Med Sci Monit, 2018. 24: p. 6313-6321.

17. He, C., et al., Overall survival and cancer-specific survival in patients with surgically resected pancreatic head adenocarcinoma: A competing risk nomogram analysis. J Cancer, 2018. 9(17): p. 3156-3167.

18. Wang, X.D., et al., Marital status independently predicts pancreatic cancer survival in patients treated with surgical resection: an analysis of the SEER database. Oncotarget, 2016. 7(17): p. 24880-7.

19. Baine, M., et al., Marital status and survival in pancreatic cancer patients: a SEER based analysis. PLoS One, 2011. 6(6): p. e21052.

20. Kattan, M.W., Nomograms. Introduction. Semin Urol Oncol, 2002. 20(2): p. 79-81.

21. Kim, S.Y., et al., Nomograms predicting survival of patients with unresectable or metastatic gastric cancer who receive combination cytotoxic chemotherapy as first-line treatment. Gastric Cancer, 2018. 21(3): p. 453-463.

22. Zheng, Z.F., et al., Development and External Validation of a Simplified Nomogram Predicting Individual Survival After RO Resection for Gastric Cancer: An International, Multicenter Study. Ann Surg Oncol, 2018. 25(8): p. 2383-2390.

23. Eguchi, H., et al., Clinicopathological Characteristics of Young Patients With Pancreatic Cancer: An Analysis of Data From Pancreatic Cancer Registry of Japan Pancreas Society. Pancreas, 2016. 45(10): p. 1411-1417.

24. Song, W., D.L. Miao, and L. Chen, Nomogram for predicting survival in patients with pancreatic cancer. Onco Targets Ther, 2018. 11: p. 539-545.

25. Li, J. and L. Liu, Overall survival in patients over 40 years old with surgically resected pancreatic carcinoma: a SEER-based nomogram analysis. BMC Cancer, 2019. 19(1): p. 726.

26. Shi, S., et al., Proposed Modification of the 8th Edition of the AJCC Staging System for Pancreatic Ductal Adenocarcinoma. Ann Surg, 2019. 269(5): p. 944-950.

27. lasonos, A., et al., How to build and interpret a nomogram for cancer prognosis. J Clin Oncol, 2008. 26(8): p. 1364-70. 
332 28. Huitzil-Melendez, F.D., et al., Advanced hepatocellular carcinoma: which staging systems best predict prognosis? J Clin Oncol, 2010. 28(17): p. 2889-95.

29. $\mathrm{Pu}, \mathrm{N}$., et al., Survival prediction in pancreatic cancer patients with no distant metastasis:

335

336 a large-scale population-based estimate. Future Oncol, 2018. 14(2): p. 165-175.

30. Mostafa, M.E., et al., Pathologic classification of "pancreatic cancers": current concepts and challenges. Chin Clin Oncol, 2017. 6(6): p. 59.

31. Springfeld, C., et al., Chemotherapy for pancreatic cancer. Presse Med, 2019. 48(3 Pt 2): p. e159-e174.

32. Stessin, A.M., J.E. Meyer, and D.L. Sherr, Neoadjuvant radiation is associated with improved survival in patients with resectable pancreatic cancer: an analysis of data from the surveillance, epidemiology, and end results (SEER) registry. Int J Radiat Oncol Biol Phys, 2008. 72(4): p. 1128-33.

33. Schulte, A., et al., Association between family cancer history and risk of pancreatic cancer. Cancer Epidemiol, 2016. 45: p. 145-150.

34. Korc, M., et al., Tobacco and alcohol as risk factors for pancreatic cancer. Best Pract Res Clin Gastroenterol, 2017. 31(5): p. 529-536. 


\section{Table 1 (on next page)}

Clinical Characteristics of Training Set and Validation Set 
1

\begin{tabular}{|c|c|c|c|c|}
\hline Characteristics & $\begin{array}{c}\text { Total } \\
4,146(100 \%)\end{array}$ & $\begin{array}{l}\text { training set } \\
2,904(70.0 \%)\end{array}$ & $\begin{array}{l}\text { validation set } \\
1,242(30.0 \%)\end{array}$ & p-value \\
\hline Race & & & & 0.005 \\
\hline White & $3,073(74.1 \%)$ & $2,192(75.5 \%)$ & $881(70.9 \%)$ & \\
\hline Black & $666(16.1 \%)$ & $450(15.5 \%)$ & $216(17.4 \%)$ & \\
\hline Others & $407(9.8 \%)$ & $262(9.0 \%)$ & $145(11.7 \%)$ & \\
\hline Gender & & & & 0.761 \\
\hline Male & $2,225(53.7 \%)$ & $1,554(53.5 \%)$ & $671(54.0 \%)$ & \\
\hline Female & $1,921(46.3 \%)$ & $1,350(46.5 \%)$ & $571(46.0 \%)$ & \\
\hline Primary site & & & & 0.205 \\
\hline Head & $1,911(46.1 \%)$ & $1,355(46.7 \%)$ & $556(44.8 \%)$ & \\
\hline Body & $473(11.4 \%)$ & $327(11.3 \%)$ & $146(11.8 \%)$ & \\
\hline Tail & $791(19.1 \%)$ & $566(19.5 \%)$ & $225(18.1 \%)$ & \\
\hline Others & $971(23.4 \%)$ & $656(22.6 \%)$ & $315(25.4 \%)$ & \\
\hline Grade & & & & 0.393 \\
\hline High differentiated & $1,272(30.7 \%)$ & $909(31.3 \%)$ & $363(29.2 \%)$ & \\
\hline Low differentiated & $744(17.9 \%)$ & $520(17.9 \%)$ & $224(18.0 \%)$ & \\
\hline Unknown & $2,130(51.4 \%)$ & $1,475(50.8 \%)$ & $655(52.7 \%)$ & \\
\hline Pathological types & & & & 0.251 \\
\hline Ductal adenocarcinoma & $2,820(68.0 \%)$ & $1,991(68.6 \%)$ & $829(66.7 \%)$ & \\
\hline Non-ductal adenocarcinoma & $1,326(32.0 \%)$ & $913(31.4 \%)$ & $413(33.3 \%)$ & \\
\hline AJCC & & & & 0.833 \\
\hline I & $418(10.1 \%)$ & $297(10.2 \%)$ & $121(9.7 \%)$ & \\
\hline II & $1,098(26.5 \%)$ & $775(26.7 \%)$ & $323(26.0 \%)$ & \\
\hline III & $321(7.7 \%)$ & $228(7.9 \%)$ & $93(7.5 \%)$ & \\
\hline IV & $2,309(55.7 \%)$ & $1,604(55.2 \%)$ & $705(56.8 \%)$ & \\
\hline Surgery & & & & 0.256 \\
\hline No & $2,593(62.5 \%)$ & $1,800(62.0 \%)$ & $793(63.8 \%)$ & \\
\hline Yes & $1,553(37.5 \%)$ & $1,104(38.0 \%)$ & $449(36.2 \%)$ & \\
\hline
\end{tabular}

2 
Table 2 (on next page)

Univariate and multivariate COX analysis of OS in training set 
1

\begin{tabular}{|c|c|c|c|c|}
\hline \multirow[t]{2}{*}{ Characteristics } & \multirow{2}{*}{$\begin{array}{c}\text { Univariate analysis } \\
\text { p-value }\end{array}$} & \multicolumn{3}{|c|}{ Multivariate analysis } \\
\hline & & $\mathrm{HR}$ & $95 \% \mathrm{CI}$ & p-value \\
\hline \multirow[t]{4}{*}{ Race } & 0.028 & & & 0.041 \\
\hline & & Reference & & \\
\hline & & 1.15 & $1.03-1.28$ & 0.016 \\
\hline & & 0.97 & $0.84-1.12$ & 0.670 \\
\hline \multirow[t]{3}{*}{ Gender } & $<0.001$ & & & 0.019 \\
\hline & & Reference & & \\
\hline & & 0.91 & $0.83-0.98$ & 0.019 \\
\hline Primary site & $<0.001$ & NA & & \\
\hline Head & & & & \\
\hline Body & & & & \\
\hline Tail & & & & \\
\hline Others & & & & \\
\hline Hich difforentioted & $<0.001$ & & & $<0.001$ \\
\hline High differentiated & & Reference & & \\
\hline Low differentiated & & 1.59 & $1.40-1.80$ & $<0.001$ \\
\hline Unknown & & 1.18 & $1.05-1.31$ & 0.004 \\
\hline Pathological types & $<0.001$ & & & $<0.001$ \\
\hline Ductal adenocarcinoma & & Reference & & \\
\hline Non-ductal adenocarcinoma & & 0.40 & $0.36-0.45$ & $<0.001$ \\
\hline $\mathrm{AJCC}$ & $<0.001$ & & & $<0.001$ \\
\hline I & & Reference & & \\
\hline II & & 3.05 & $2.23-3.96$ & $<0.001$ \\
\hline III & & 3.10 & $2.30-4.18$ & $<0.001$ \\
\hline IV & & 4.63 & $3.53-6.07$ & $<0.001$ \\
\hline Surgery & $<0.001$ & & & $<0.001$ \\
\hline No & & Reference & & \\
\hline Yes & & 0.43 & $0.38-0.50$ & $<0.001$ \\
\hline
\end{tabular}

2 


\section{Table 3(on next page)}

Univariate and multivariate competing analysis of CSS in training set 
1 Table 3. Univariate and multivariate competing analysis of CSS in training set

\begin{tabular}{|c|c|c|c|c|}
\hline & \multirow{2}{*}{$\begin{array}{c}\text { Univariate analysis } \\
\text { p-value }\end{array}$} & \multicolumn{2}{|c|}{ Multivariate analysis } & \multirow[b]{2}{*}{ p-value } \\
\hline & & SHR & $95 \% \mathrm{CI}$ & \\
\hline Race & & NA & & \\
\hline White & & $\underline{\text { Reference }}$ & & \\
\hline Black & 0.210 & $\underline{1.07}$ & $\underline{0.94-1.21}$ & $\underline{0.30}$ \\
\hline Others & 0.260 & $\underline{1.06}$ & $\underline{0.90-1.24}$ & $\underline{0.51}$ \\
\hline Gender & & $\mathrm{NA}$ & & \\
\hline Male & & $\underline{\text { Reference }}$ & & \\
\hline Female & 0.78 & $\underline{1.02}$ & $\underline{0.94-1.12}$ & $\underline{0.63}$ \\
\hline \multicolumn{5}{|l|}{ Primary site } \\
\hline Head & & Reference & & \\
\hline Body & 0.046 & 1.01 & $0.88-1.17$ & $0.8 \underline{6} 7$ \\
\hline Tail & 0.091 & 1.09 & $0 . \underline{9686-1.24}$ & $0 . \underline{18} 21$ \\
\hline Others & 0.750 & 0.87 & $0.77-0.99$ & 0.037 \\
\hline \multicolumn{5}{|l|}{ Grade } \\
\hline High differentiated & & Reference & & \\
\hline Low differentiated & 0.024 & 1.09 & $0.96-1.23$ & 0.178 \\
\hline Unknown & $<0.001$ & 1.098 & $0.97-1.21$ & $0.1 \underline{4} 8$ \\
\hline \multicolumn{5}{|l|}{ Pathological types } \\
\hline Ductal adenocarcinoma & & Reference & & \\
\hline Non-ductal adenocarcinoma & $<0.001$ & 0.57 & $0.50-0.65$ & $<0.001$ \\
\hline \multicolumn{5}{|l|}{ AJCC } \\
\hline I & & Reference & & \\
\hline II & 0.063 & 1.22 & $0.9 \underline{9} 8-1.5 \underline{1} \theta$ & 0.072 \\
\hline III & $<0.001$ & 1.26 & $0.9 \underline{8} 9-1.61$ & $0.06 \underline{9} z$ \\
\hline IV & $<0.001$ & 1.53 & $1.22-1.9 \underline{3} z$ & $<0.001$ \\
\hline \multicolumn{5}{|l|}{ Surgery } \\
\hline No & & Reference & & \\
\hline Yes & $<0.001$ & 0.77 & $0.68-0.876$ & $<0.001$ \\
\hline
\end{tabular}

2 
Figure 1

Flowchart of patient selection

Detailed selection of PC patients in 2004-2015 from SEER database.

SEER database 2004-2015

Pancreatic cancer $\mathrm{N}=130,458$

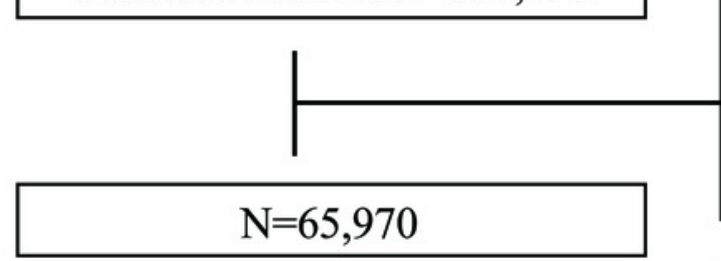

Age at diagnosed $\geq 50$ years old

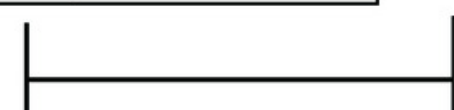

a. Multi-primary cancers

b. AJCC stage NOS, N/A or unknown

a. Age, race, surgery unknown

b. Survival time unknown and less than 1 month

c. Diagnosis by autopsy and death certificate

d. Pathological diagnosis unknown

$\mathrm{N}=47,081$

$$
\begin{gathered}
\text { Patients }<50 \text { years old } \\
\mathrm{N}=4,146
\end{gathered}
$$


Figure 2

Nomogram for predicting OS and CSS of young patients with PC.

A. Nomogram for predicting 1-, 3- and 5-year OS for young patients with PC; B. Nomogram for predicting 1-, 3- and 5-year CSS for young patients with PC 
A

Points

Gender

Grade

Race

Pathological.types

Surgery

AJCC.stage

Total Points

1-year OS

3-years OS

5-years OS

B

Points

Primary.site

Pathological.types

AJCC.stage

Surgery

Total Points

1-year CSS

3-years CSS

5-years CSS
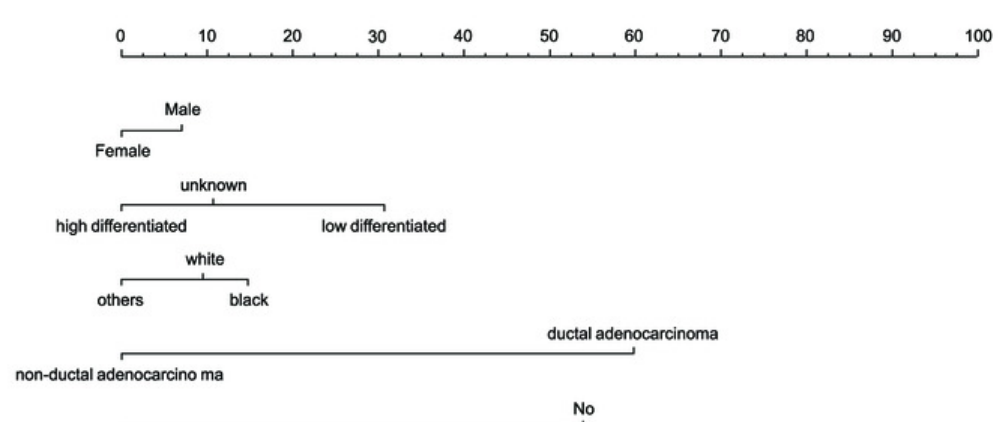

Yes

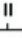

III

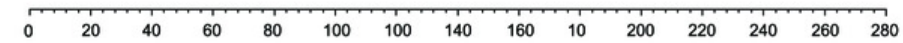

\begin{tabular}{lllllllll}
\hline 0.9 & 0.8 & 0.7 & 0.6 & 0.5 & 0.4 & 0.3 & 0.2 & 0.1
\end{tabular}

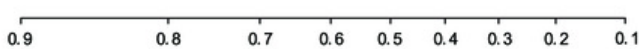

\begin{tabular}{llllllll}
\hline 0.8 & 0.7 & 0.6 & 0.5 & 0.4 & 0.3 & 0.2 & 0.1
\end{tabular}

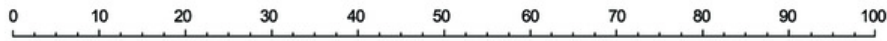

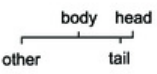

ductal adenocarcinoma

non-ductal adenocarcinoma

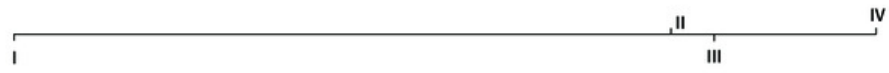

No
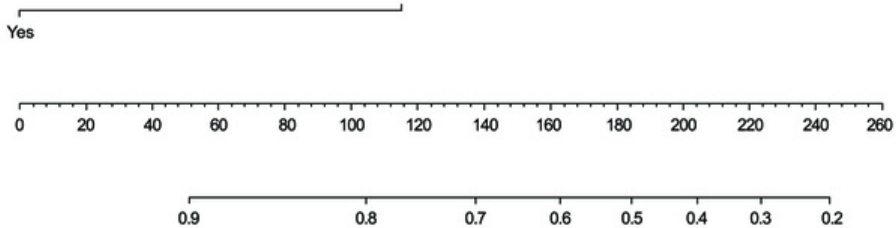

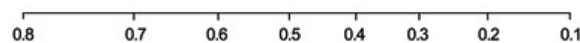

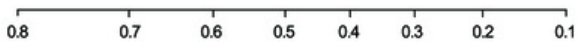


Figure 3

Calibration curves for 1-, 3- and 5-year OS in training set and validation set.

A. Calibration curves for 1-year OS in training set; B. Calibration curves for 3-year OS in training set; C. Calibration curves for 5-year OS in training set; D. Calibration curves for 1year OS in validation set; E. Calibration curves for 3-year OS in validation set; F. Calibration curves for 5-year OS in validation set.
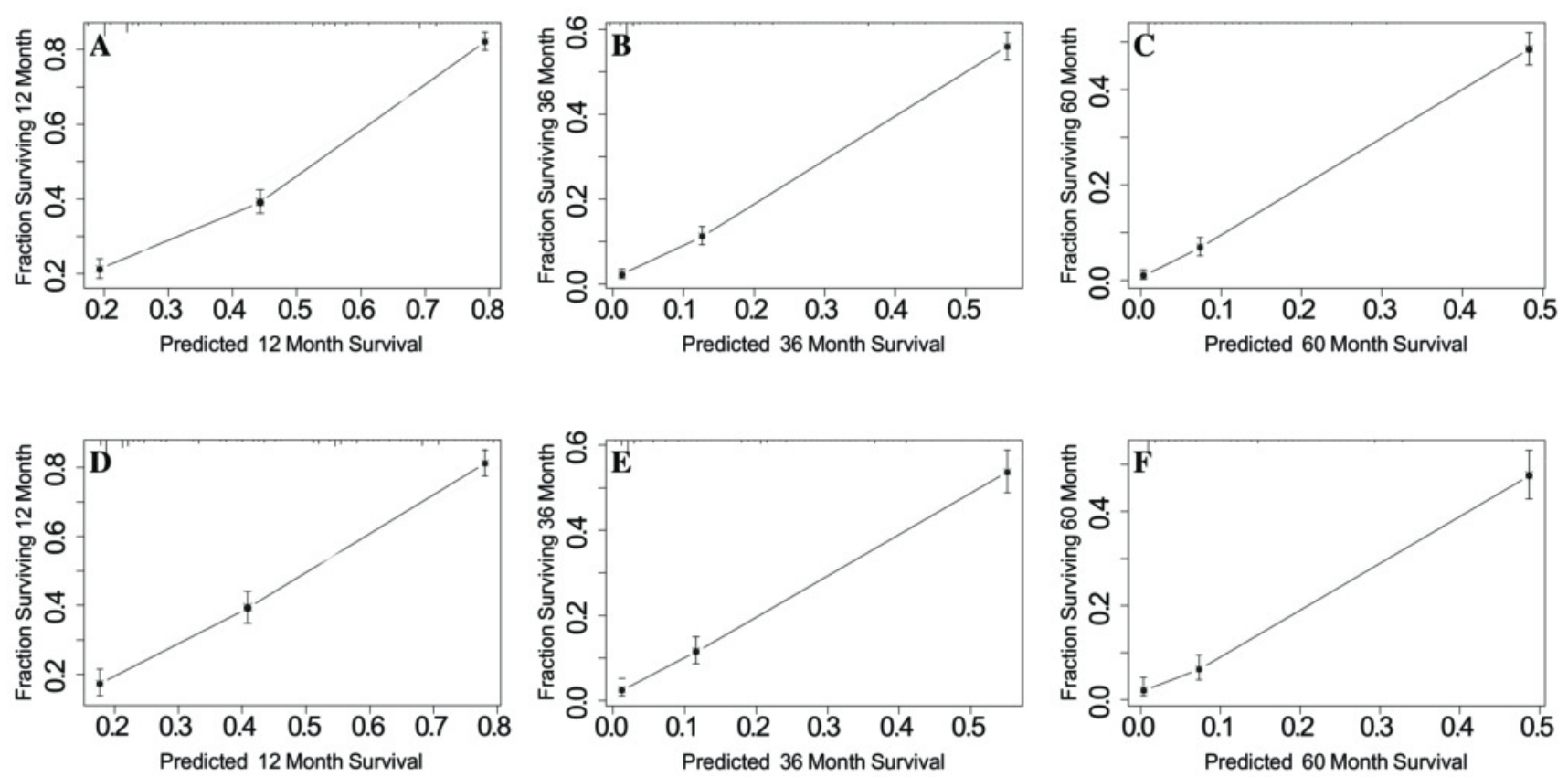
Figure 4

Calibration curves for 1-, 3- and 5-year CSS in training set and validation set.

A. Calibration curves for 1-year CSS in training set; B. Calibration curves for 3-year CSS in training set; C. Calibration curves for 5-year CSS in training set; D. Calibration curves for 1year CSS in validation set; E. Calibration curves for 3-year CSS in validation set; F. Calibration curves for 5-year CSS in validation set.
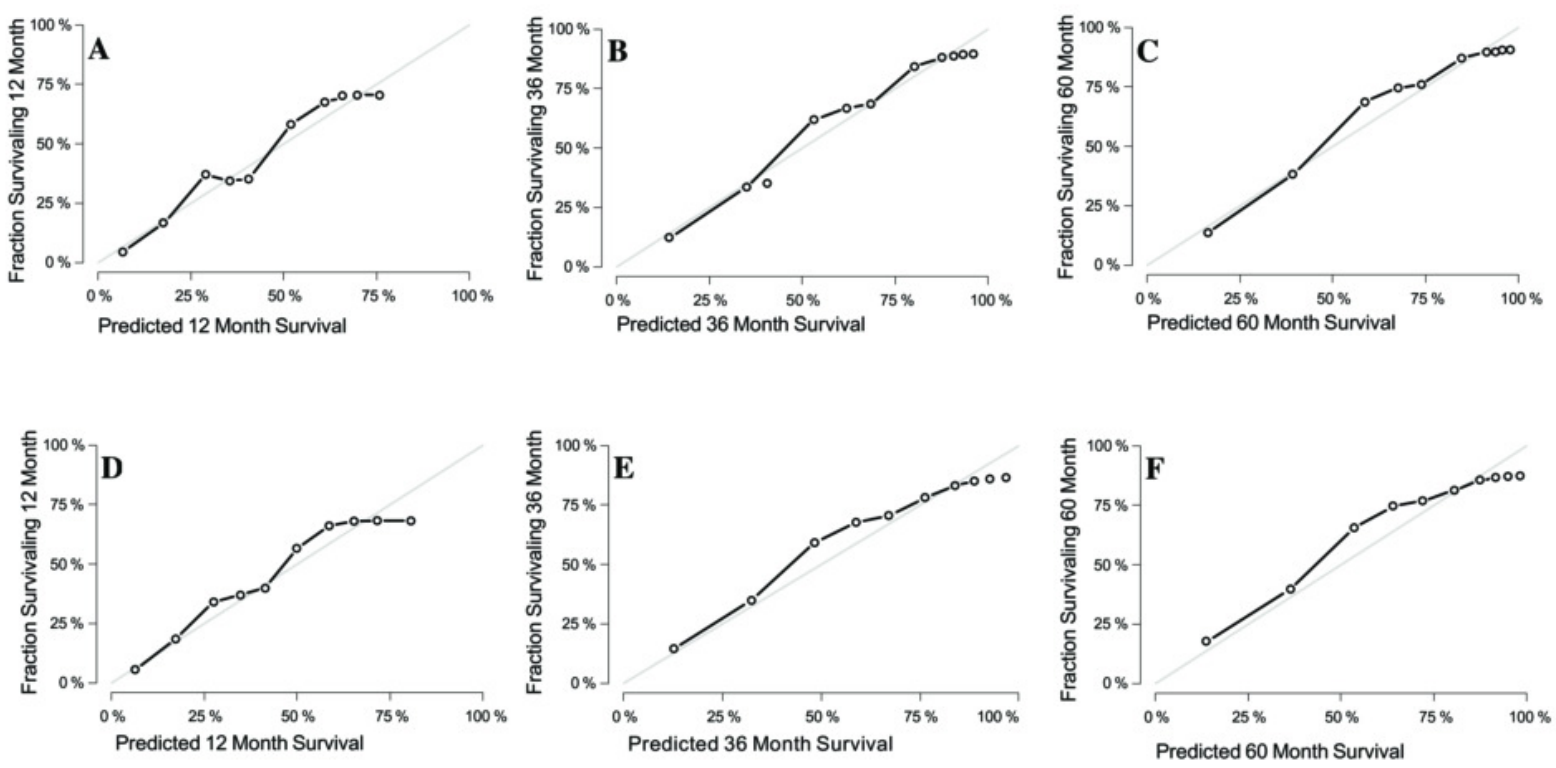